\title{
Research on Pre-processing for Pointer Meter Automatic Recognition
}

\author{
Pei Liang ${ }^{1,2}$ a , Li Wenqing ${ }^{1,2, b}$, Wang Bo ${ }^{1,2, c}$, Liu Shixuan ${ }^{1,2, d}$ \\ ${ }^{1}$ Shandong Provincial Key Lab of Ocean Environment Monitoring Technology, Qingdao, China \\ ${ }^{2}$ Institute of Oceanographic Instrumentation, Shandong Academy of Science, Qingdao, China \\ apeiliang2002@163.com, ${ }^{\mathrm{b}}$ livenson@163.com, ${ }^{\mathrm{c}}$ bob80.wang@hotmail.com,, ${ }^{\mathrm{d}} 321$ _go@sohu.com
}

Key words: Image grizzle; Median filter; Threshold segmentation; Gray stretch; Bigness method Abstract. The accurate detection of the pointer position is the key of the automatic pointer meter reading. Appropriate image preprocessing is the basis of the precise location of the scale zone and pointer on the dial. In this paper, the images are preprocessed by image grizzle, median filter and image binarization. In order to improve the quality of the image segmentation, an improved bigness method based on gray stretch is adopted in the threshold segmentation. Experiments results show that effective thresholds are obtained, the useful information is successfully reserved and satisfactory segmentation results of the pointer meter images are achieved.

\section{Introduction}

Pointer meter is widely used in labs and production for its simple structure and convenience. With the development of digital electronic technology, some of the pointer meters have been replaced by digital ones. Digital meters have high precision and are convenient in data reading. But when the signal to be measured changes rapidly or fluctuates, its readings will also change rapidly, so it is not easy to be read. Moreover, if the display time interval is controlled, details of the changing process will be ignored. At this point, pointer meters have advantages over digital ones. Pointer meters can directly reflect the dynamic changing and trends of the value measured. Therefore, point meters still play an irreplaceable role [1].

But for pointer meters, manual operation and eye measure are usually used in the real application. So some subjective factors, such as the tester's operation experience, operation habit and mental state, may influence the data reading. And problems like high labor intensity, low production efficiency and big testing error also exists.

In recent years, the automatic pointer meter recognition becomes a hot topic, based on which the efficiency and precision of data reading can be greatly improved [2]. And the main purpose for pointer meter automatic recognition is the accurate detection of the pointer position. And appropriate image preprocessing should be firstly carried out for the precise location of the scale zone and pointer on the dial.

For the automatic point meter recognition, the main purpose of the image preprocessing is to emphasize the image characteristics users are interested in and eliminate the useless information. In real applications, there will be different kind of noise or interference signals during data acquisition. This makes the detection of dial image and the recognition of scale zone and pointer difficult. So, the preprocessing of the instrument images is important.

In this paper, the images are preprocessed by image gray scaling, median filter and image binarization. The image preprocessing flowchart is shown in Fig.1.

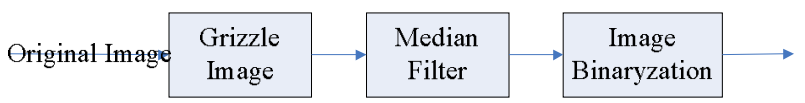

Fig.1 Image preprocessing process

\section{Gray scaling of instrument images}

Gray images contain only brightness information and no color information. The true color 
meter images contain a large number of color information and need to be converted to gray images for further processing and recognition.

In the transform of color images to gray images, the $R 、 G 、 B$ color components of each pixel are considered, and the corresponding grey value can be computed with the following formula $^{[3]}$ :

$$
H=0.2989 \cdot R_{+} 0.5870^{\bullet} G_{+} 0.1140 \cdot B
$$

Here, $R 、 G 、 B$ respectively represent the color value of red, green, blue, and $H$ is the gray value after being converted. Fig. 2 is the gray image after being gray-scaled.

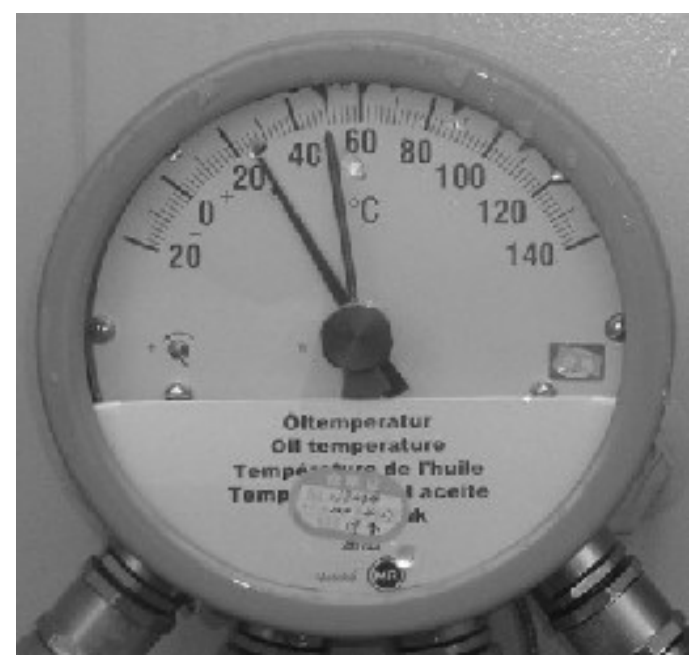

Fig.2 The gray image after being gray-scaled

\section{Smoothing of Instrument Images}

The images are often affected by the noise from imaging equipment, transmission equipment and external environment during the process of data acquisition, transmission and transformation. So the actual images generally contain noise, which will affect the detection of the edge points. Therefore, it is necessary to eliminate the various noises before the instrument images recognition The operation of removing the noise from images is called smoothness.

In this paper, the figure window with the size of $3^{*} 3$ is used to realize the 9 -points median filter. Median filter is a nonlinear processing technology which can be used to restrain the noise of image and not make edge blurred. Its main idea is to replace one point value of the digital image or digital sequence with the median value of an adjacent domain [4], which can eliminate the isolated noise points.

Median filter is a sliding window containing odd number of points, in which the central point grey value is replaced by the median value of all points. Its impulse response is zero. So it have great advantage in suppressing impulsive noises. Using median filter, the noise can be efficiently suppressed, so the details of image can be retained. Fig.3 is the instrument image processed by median filter. 


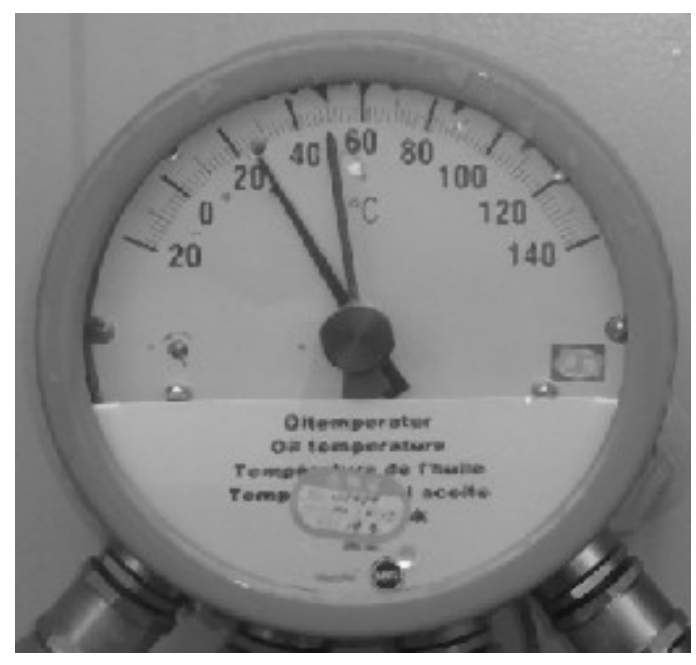

Fig.3 Instrument image after a median filter

From Fig.3, we can see that the random noise is efficiently suppressed and the edges were still sharp. It can be concluded that Median filter can maintain the target image information, optimize image quality, remove medium-sized noise and maintain the Laplace domain shape gray.

\section{Threshold segmentation of pointer meter images}

After image grey scaling and median filter, image binarization should be carried out, that is, to segmemt the image with the threshold determined by some criteria, and we call threshold segmentation here. Based on above the standardization image can be obtained.

Threshold segmentation is a technology based on regional image segmentation. Its basic principle is: firstly, determine a grey-scale threshold in the range of the image grey scale, then compare grey scale of each pixel with the threshold value and divide the corresponding pixels into two categories. The result of threshold segmentation is to convert grayscale image into binary image. Supposing the original image is $f(x, y)$, the threshold $\mathrm{T}$ should be determined according to certain standards, the binary image after threshold segmentation can be expressed as formula (2) [5]:

$$
g(x, y)=\left\{\begin{array}{lr}
0 & f(x, y)<T \\
255 & f(x, y)<T
\end{array}\right.
$$

The basic requirements for image binarization are the following: image after binarization can accurately represent the original image. The determination of the threshold $\mathrm{T}$ is the key of threshold segmentation. In this paper, the iterative method, maximum entropy method and bigness method are used to determine threshold $\mathrm{T}$ of the instrument image. In order to enhance the quality of the image segmentation, an improved bigness method based on gray stretch for threshold segmentation was adopted.

Iteration method. Iteration method is based on approximation principle [6]. It can be described as the following: firstly, choose an approximate value as the original threshold value; then divide the image to obtain sub-images and chose a new threshold value according to the characteristics of sub-images; use new threshold value to divide the image. After several cycles, the number of error pixesl in image segmentation can be greatly reduced.

The optimal threshold value can be obtained using the iteration method, based on which the influence of noise can be efficiently suppressed. But iteration method has high requirements for the brightness of the image. But to images with fluctuating gray values, the segmentation result is not so good. 


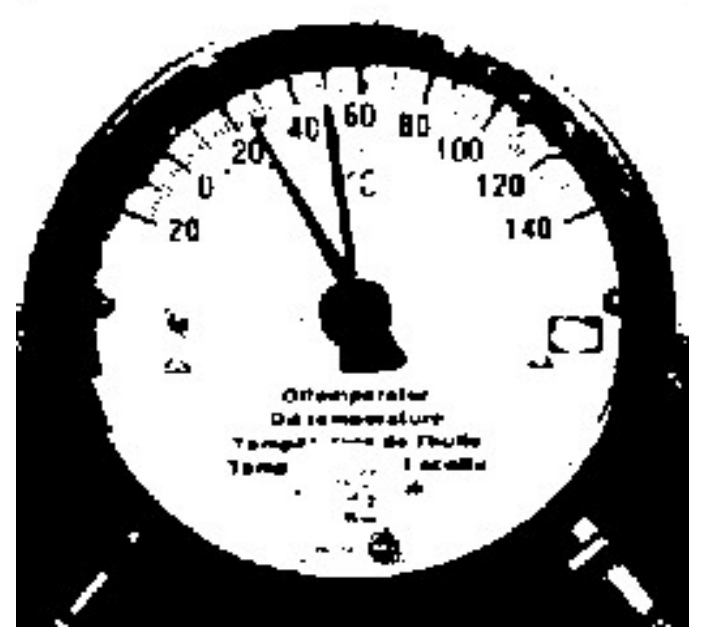

Fig.4 Threshold segmentation effect based on iteration method

Fig.4 is the threshold segmentation result based on iteration method. As shown in this figure, the image segmentation threshold value computed using iteration is too small, some useful information of the target object was lost, and part of the background object information still remained. So the segmentation effect is far from perfect.

Maximum entropy method. At the beginning of 1980s, people considered using the concept of entropy in information theory to decide the threshold value. Entropy is a form of statistical properties of images, which indicates the information quantity an image contains. The idea of maximum entropy method is that the ideal threshold value of image segmentation should make the information quantity of the image to be the maximum, which means the entropy is maximum [7-9].

Like Otsu method, maximum entropy method need to calculate the function value to find out the maximum of the corresponding gray scale, and the corresponding gray scale is the ideal threshold.

The one-dimension maximum entropy method has good segmentation effect and is simple, it is a representative method. But one-dimension histogram can't indicate the image's regional spatial information. So when the image is influenced by factors like noise, one-dimension maximum entropy can't perform well. And the two-dimension maximum entropy method based on two-dimension histogram has good anti-noise property, but its computation cost is too large, which makes it not so practical in real applications. Fig.5 is the threshold segmentation effect based on maximum entropy method.

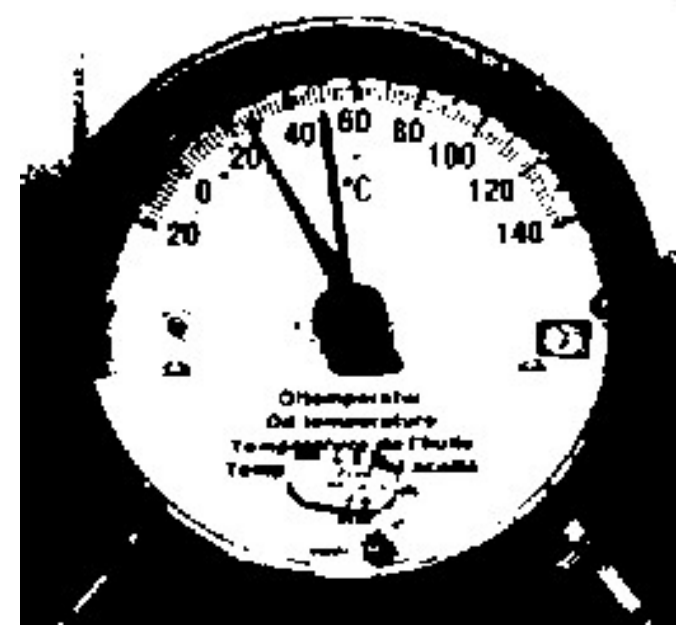

Fig.5 Threshold segmentation effect based on maximum entropy method 
Bigness method. Bigness method, named as OTUS, was proposed by Otus in 1978 [10]. The basic idea of bigness method is the following: take a gray scale and use it as the threshold and divide the image into two categories, that is, large grayscale and small grayscale; then calculate the number of pixels and then the average grayscale of the two categories, and inter-class variance; finally, adopt the grey scale value with the maximum class variance as the threshold.

Variance is the measurement of the image's grey scale homogeneous distribution. Class variance represents the difference of light and shade between the images. The larger the variance is, the bigger the difference between two images is. Here, the mistaken division of objects to background will decrease the difference. So here maximizing the class variance means to minimize the error distribution probability [11]. The threshold segmentation effect using bigness method is shown in Fig. 6.

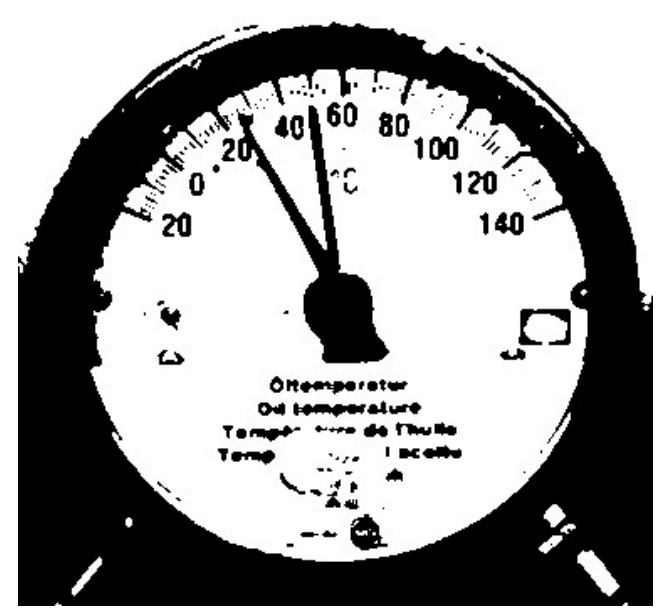

Fig. 6 Binary image using bigness method

Fig.6 indicates that the threshold obtained from bigness method is good. The segmentation quality is stable. It has good segmentation results in high SNR conditions. Its drawback is that when the grey scale difference between object and background is not obvious, big black regions will appear, and even the whole information of the image may be lost. Because it only considers the grey scale of the pixels, so has low anti-interference ability. And the threshold is used on every pixel of the image. Therefore, to the images with big grey scale gradient difference between object and background, the segmentation result is not satisfactory.

Improved bigness method. To the problems of the bigness method, an improved bigness method based on gray stretch for threshold segmentation was adopted. In this method, the gray level was stretched to amplify the gray scale difference between the foreground and background.

In gray stretch, segmental linear transformation is performed to compress the grey scale difference of the background and to increase the contrast ratio of the foreground of the image. By gray stretch, we can lower the probability of big black regions, and keep the threshold in the stretched gray range, so it is easier to process the grey scale range we are interested in.

The stretch transform function is shown in Fig.7, the threshold is between $\mathrm{x} 1$ and $\mathrm{x} 2$, which is the grayscale range we are interested in. The range of grey scale was multiplied by a stretch coefficient and the range was stretched to [y1, y2]. With stretch transform function, the grey scale difference between foreground and background can be increased and the contrast ratio too[12].

The gray stretch transform function used in this paper is the following: 


$$
f(x)\left\{\begin{array}{lc}
\frac{y_{1}}{x_{1}} x & x<x_{1} \\
\frac{y_{2}-y_{1}}{x_{2}-x_{1}}\left(x-x_{1}\right)+y_{1} & x_{1} \leq x \leq x_{2} \\
\frac{255-y_{2}}{255-x_{2}}\left(x-x_{2}\right)+y_{2} & x \geq x_{2}
\end{array}\right.
$$

Here, $(\mathrm{x} 1, \mathrm{y} 1)$ and $(\mathrm{x} 2, \mathrm{y} 2)$ are the coordinates of the turning points in figure 7.

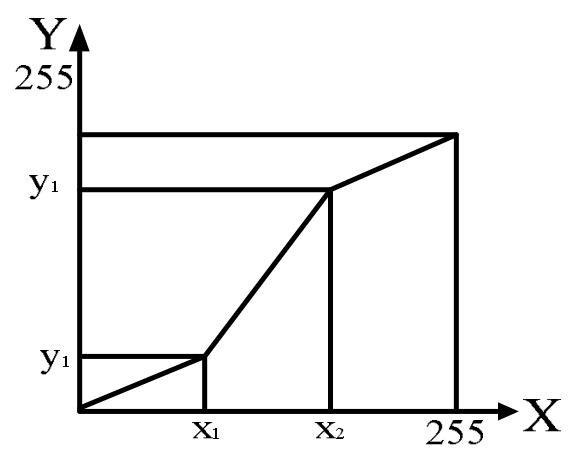

Fig. 7 Gray stretch transform function

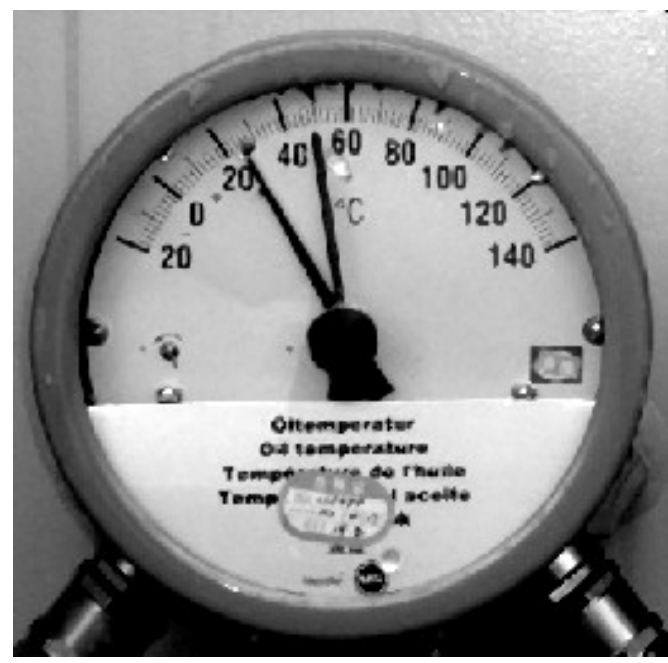

Fig. 8 The image which has been stretched

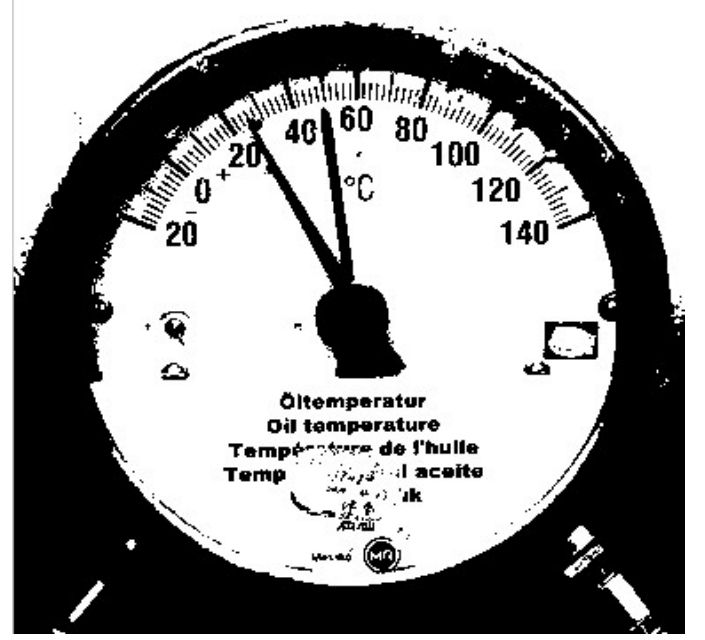

Fig. 9 Binary image using improved bigness method

Fig. 8 is a stretched image. We can see that after the image grayscale stretch the quality of the image has been improved and the grey scale difference of foreground and background was increased. In the improved bigness method, gray stretch is firstly carried out, then the bigness method is applied to get the binary image. The binarization results are shown in Fig.9, from which we can see that precise segmentation results can achieved by the improved bigness method.

\section{Conclusion}

Several threshold segmentation methods are introduced and performance comparison is carried out. The improved bigness method based on the gray stretch is adopted in the binarization of pointer meter image. The improved bigness method overcomes the drawbacks of the bigness method, and it's effective in conditions with small gray difference between the object and the background, so precise binarization segmentation images consisting to the original image can be achieved. Experiment results show that the improved bigness method can produce precise thresholds, can 
eliminate the unwanted big black regions, and can reserve the useful information. With the improved bigness method, satisfactory segmentation results of the pointer meter images were achieved, and this is the foundation for the further automatic point meter recognition

\section{Acknowledgements}

This work was financially supported by special funds for scientific research projects of Marine public welfare industry: (201405022-2).

\section{References}

[1] Shiwei CHEN, Shiping LI, The Design and Implementation on the automatic recognition of Pointer Voltmeter Precision System [J]. Computer Measurement and Control, 2005.13(11):1193.

[2] F Correa Alegria, A Cruzz, Automatic Calibration of Analog and Digital Measuring Instruments Using Computer Vision [J]. IEEE Transaction on Instrumentation and Measurement, 2000.49 (1): 94-99.

[3] Shihai DONG, Programming Guide of Image Formats. Tsinghua university, Beijing. 1994.

[4] Kenneth R.Castleman, Digital Image Processing, USA, Prentice Hall. 1996.

[5] Yujin ZHANG, Image Science Series - Image Segmentation. Science Press. 2001.

[6] Xiaofeng HU, Practical Case Selection of Visual C++/MATLAB Processing and Identify. People's Post Press. 2004.

[7] Pun T. A New Method for Gray-level Picture Thresholding Using the Entropy of Histogram. Signal Processing, 1980. 2: 223-23[8] KittlerJ, Illingworth J, Minimum Error Thresholding. Pattern Recognition, 1986.19(1):41-47.

[9] Kapur J N, Sahoo P K, Wong A K C, A New Method for Gray-level Picture Thresholding Using the Entropy of the Histogram [J]. Computer Vision, Graphics, and Image Processing, 1985.29(3): 273-285.

[10] Ostu N A, Threshold Selection Method from Gray-Level Histograms [J]. IEEE Trans. On System, Man, and Cybernetics, 1979.9(1): 62-66.

[11] Junzhe LV, Research and Realization of Image Binaryzation. Science and Technology Information Development and Economic, 2004.14(12): 266-267.

[12] Jiangtao FAN, The Research and Application of Meter Recognition Based on Engineer Vision. Guangdong University of Technology, Guangdong, 2008. p.22-24. 